Supporting Information

\title{
Combined Signal Amplification Using a Propagating Cascade Reaction and a Redox Cycling Reaction for Sensitive Thyroid- Stimulating Hormone Detection
}

Seonhwa Park, ${ }^{\dagger}$ Jihyeon Kim, ${ }^{\dagger}$ Seheon Kim,${ }^{\dagger}$ Gyeongho Kim, ${ }^{\dagger}$ Nam-Sihk Lee, ${ }^{\ddagger}$ Young Ho Yoon, ${ }^{\star}$ and Haesik Yang ${ }^{*} \dagger$

†Department of Chemistry and Chemistry Institute for Functional Materials, Pusan

National University, Busan 46241, Korea

‡EONE Laboratories, Incheon 22014, Korea 


\section{Table of Content}

Figure S-1. Schematic diagrams of sandwich-type immunoassay using (a) ecarinprothrombin cascade reaction and (b) thrombin label and EC redox cycling.

Figure S-2. (a) Chronocoulograms in the absence of EC redox cycling obtained (at 0.15 $\mathrm{V}$ ) at BSA/avidin/rGO/ITO electrodes in PBS (pH 7.4) containing (i) $0.1 \mathrm{mM}$ FPK-AP, (ii) (i) and $10 \mu \mathrm{g} / \mathrm{mL}$ prothrombin, (iii) (i) and $1 \mu \mathrm{g} / \mathrm{mL}$ ecarin and (iv) (ii) and $1 \mu \mathrm{g} / \mathrm{mL}$ ecarin. (b) Chronocoulograms in the presence of EC redox cycling obtained (at $0.15 \mathrm{~V}$ ) at BSA/avidin/rGO/ITO electrodes in PBS (pH 7.4) containing (i) $0.1 \mathrm{mM}$ FPK-AP and $2.0 \mathrm{mM} \mathrm{NADH}$, (ii) (i) and $10 \mu \mathrm{g} / \mathrm{mL}$ prothrombin, (iii) (i) and $1 \mu \mathrm{g} / \mathrm{mL}$ ecarin, (iv) (ii) and $1 \mu \mathrm{g} / \mathrm{mL}$ ecarin and (v) (i) and $1 \mu \mathrm{g} / \mathrm{mL}$ thrombin. Chronocoulograms obtained after an incubation period for $30 \mathrm{~min}$ at $37^{\circ} \mathrm{C}$.

Figure S-3. (a) Chronocoulograms obtained (at $0.15 \mathrm{~V}$ ) using (a) Figure S-1a and (b) Figure S-1b (in artificial serum containing different concentrations of TSH) after an incubation period of $30 \mathrm{~min}$ at $37^{\circ} \mathrm{C}$ in a mixed solution of PBS containing (a) $0.1 \mathrm{mM}$ FPK-AP and $10 \mu \mathrm{g} / \mathrm{mL}$ prothrombin and (b) $0.1 \mathrm{mM}$ FPK-AP and $2.0 \mathrm{mM} \mathrm{NADH}$.

Figure S-4. Calibration plots of the charge measured at $100 \mathrm{~s}$ in the chronocoulograms of (a) Figure S-3a obtained using Figure S-1a and (b) Figure S-3b obtained using Figure S-1b. All experiments were conducted using 3 different electrodes for each sample. The data were subtracted from the mean value at a concentration of zero determined from seven measurements. The dashed line corresponds to three times the standard deviation (SD) of the charge at zero concentration. The error bars represent the SD of three measurements. 
(a)

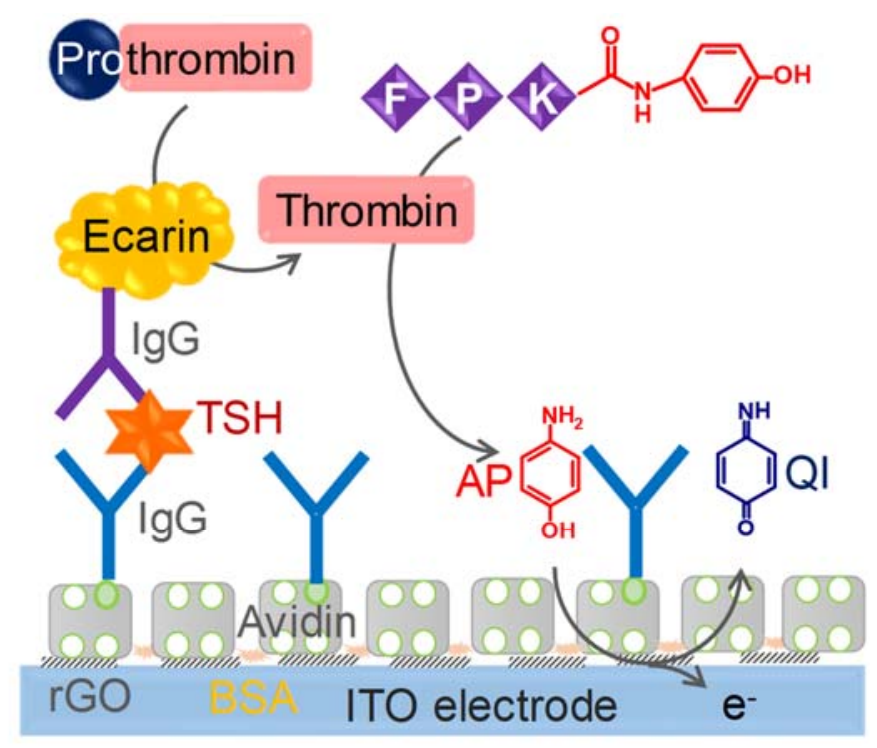

(b)

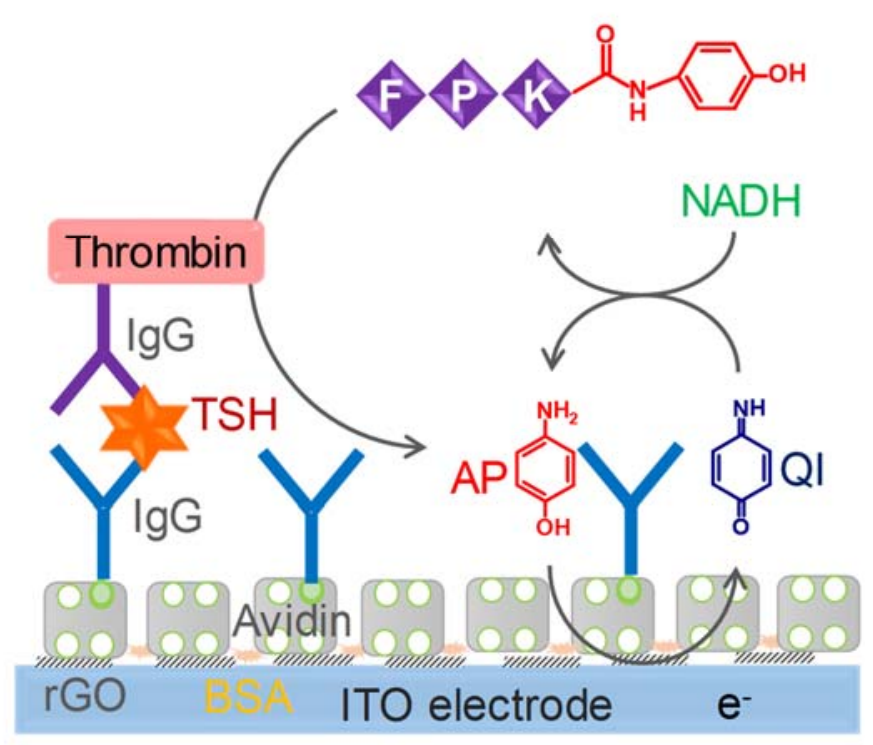

Figure S-1. Schematic diagrams of sandwich-type immunoassay using (a) ecarinprothrombin cascade reaction and (b) thrombin label and EC redox cycling. 

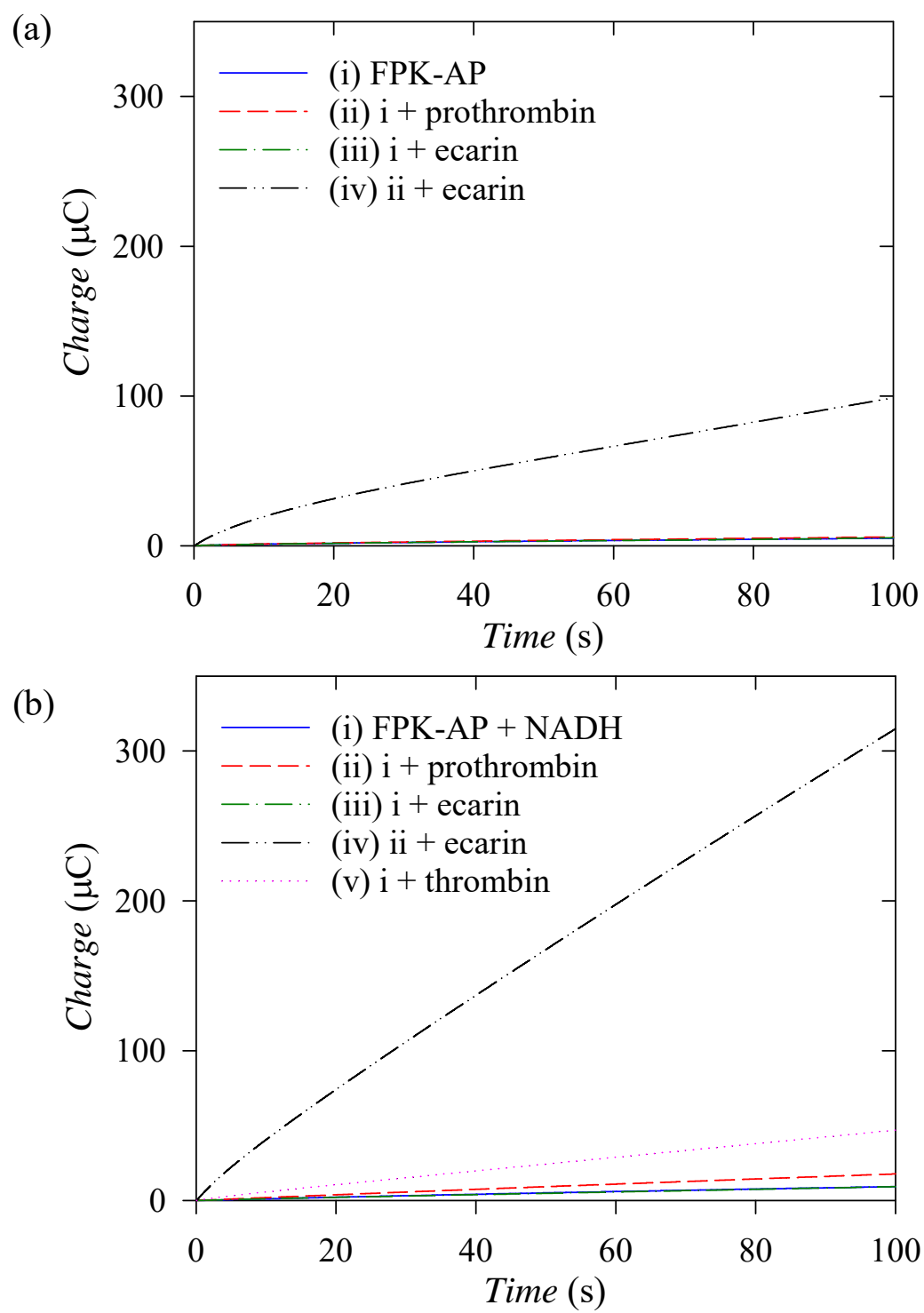

Figure S-2. (a) Chronocoulograms in the absence of EC redox cycling obtained (at 0.15 $\mathrm{V}$ ) at BSA/avidin/rGO/ITO electrodes in PBS (pH 7.4) containing (i) $0.1 \mathrm{mM}$ FPK-AP, (ii) (i) and $10 \mu \mathrm{g} / \mathrm{mL}$ prothrombin, (iii) (i) and $1 \mu \mathrm{g} / \mathrm{mL}$ ecarin and (iv) (ii) and $1 \mu \mathrm{g} / \mathrm{mL}$ ecarin. (b) Chronocoulograms in the presence of EC redox cycling obtained (at $0.15 \mathrm{~V}$ ) at BSA/avidin/rGO/ITO electrodes in PBS (pH 7.4) containing (i) $0.1 \mathrm{mM}$ FPK-AP and $2.0 \mathrm{mM}$ NADH, (ii) (i) and $10 \mu \mathrm{g} / \mathrm{mL}$ prothrombin, (iii) (i) and $1 \mu \mathrm{g} / \mathrm{mL}$ ecarin, (iv) (ii) and $1 \mu \mathrm{g} / \mathrm{mL}$ ecarin and (v) (i) and $1 \mu \mathrm{g} / \mathrm{mL}$ thrombin. Chronocoulograms obtained after an incubation period for $30 \mathrm{~min}$ at $37^{\circ} \mathrm{C}$. 

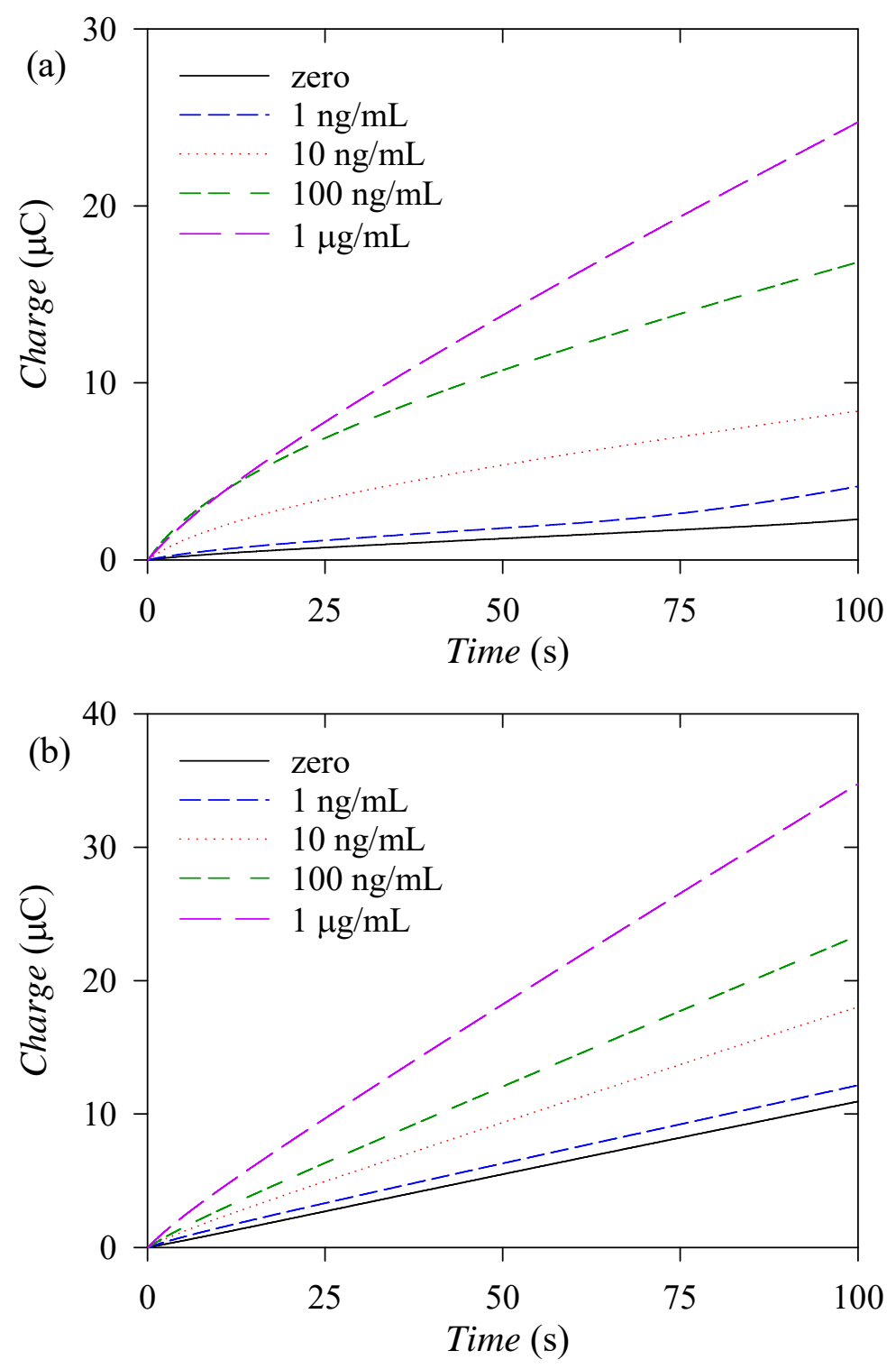

Figure S-3. (a) Chronocoulograms obtained (at $0.15 \mathrm{~V}$ ) using (a) Figure S-1a and (b) Figure S-1b (in artificial serum containing different concentrations of TSH) after an incubation period of $30 \mathrm{~min}$ at $37^{\circ} \mathrm{C}$ in a mixed solution of PBS containing (a) $0.1 \mathrm{mM}$ FPK-AP and $10 \mu \mathrm{g} / \mathrm{mL}$ prothrombin and (b) $0.1 \mathrm{mM}$ FPK-AP and $2.0 \mathrm{mM}$ NADH. 

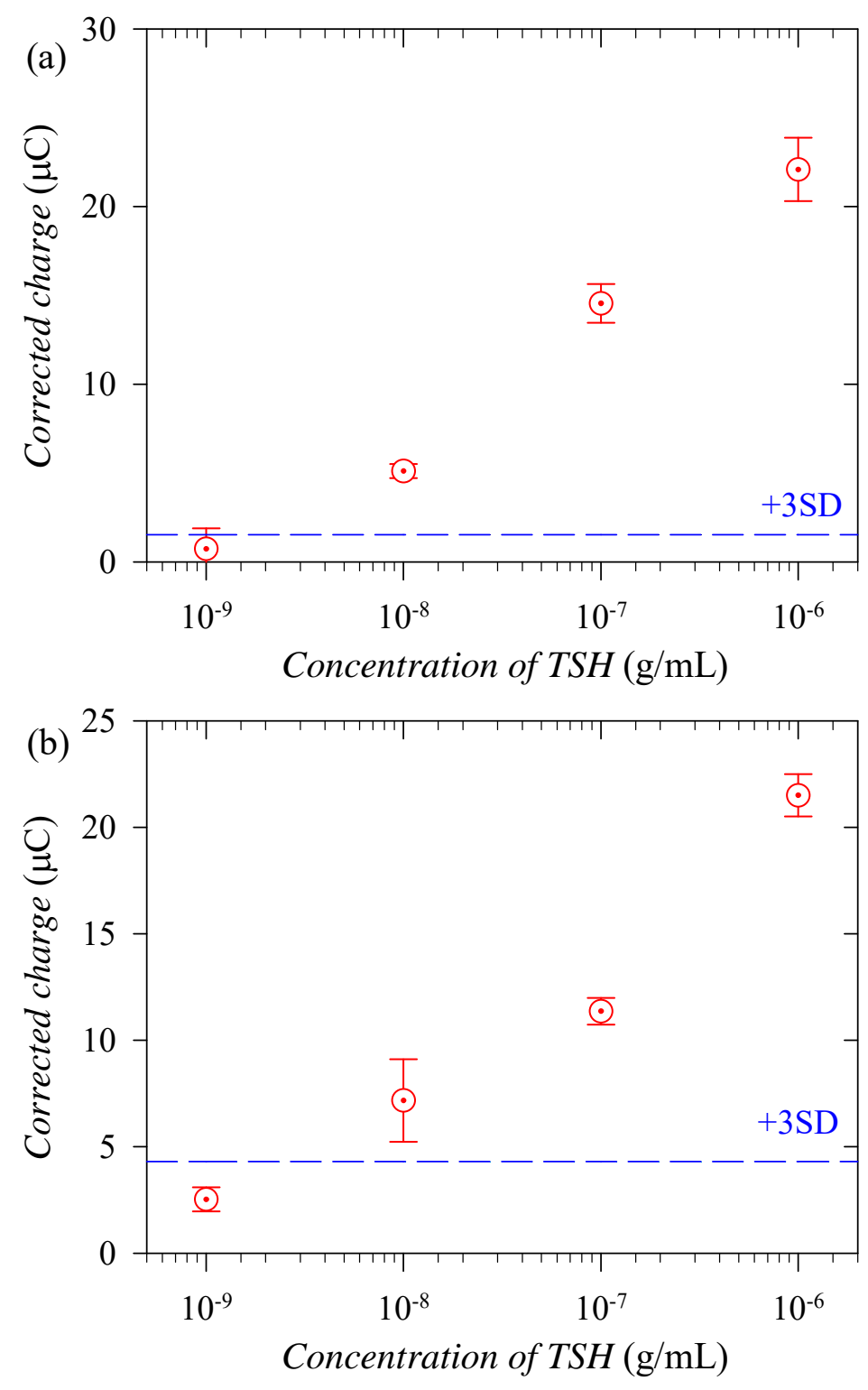

Figure S-4. Calibration plots of the charge measured at $100 \mathrm{~s}$ in the chronocoulograms of (a) Figure S-3a obtained using Figure S-1a and (b) Figure S-3b obtained using Figure S-1b. All experiments were conducted using 3 different electrodes for each sample. The data were subtracted from the mean value at a concentration of zero determined from seven measurements. The dashed line corresponds to three times the standard deviation (SD) of the charge at zero concentration. The error bars represent the SD of three measurements. 